UMDHEP-93-201

ISJ-5079

May 1993

\title{
Effective Lagrangian Approach to Electroweak Baryogenesis: higgs mass limit and electric dipole moments of fermion
}

\author{
X. Zhang \\ Department of Physics \& Astronomy, University of Maryland, \\ College Park, MD 20742 \\ and \\ B.-L. Young \\ Department of Physics \& Astronomy, Iowa State University, \\ Ames, Iowa 50011
}

\begin{abstract}
A natural solution to the hierarchy problem of the standard model is to assume new physics to appear at the TeV scale. We parametrize the effects of this new physics in terms of effective lagrangian and examine its impacts on electroweak baryogenesis. We point out that with such an effective lagrangian successful electroweak baryogenesis implies: i) Higgs boson lies within the reach of LEP II; ii) electric dipole moments of electron and neutron are detectable in the near future.
\end{abstract}


Recent experimental data from LEP and SLC has provided remarkable checks of the validity of the standard model[1]. The data is in agreement with the theoretical prediction of the model to a level which is better than one percent[2]. Even though the standard model is perfectly consistent with all evidence gathered to date, it raises as many questions as it answers. The so-called hierarchy problem is one of them, and it is based on the observation that the quadratic divergence of the Higgs sector makes it difficult to explain a widely separated hierarchy between the Fermi scale and a very high scale of new physics, $\Lambda$. Obviously, the hierarchy problem is solved if $\Lambda$ is also around the Fermi scale. In this case, one would expect new physics beyond the standard model to appear at the $\mathrm{TeV}$ scale.

Another indication for the possible existence of new physics is from the current understanding of the baryon asymmetry of the universe. In Sakharov's original proposal[3], three ingredients are required: a) baryon number violation; b) $\mathrm{CP}$ and $\mathrm{C}$ violation; c) thermodynamic nonequilibrium. Indeed the three conditions can be satisfied[4] in the standard model. Unfortunately the net matter- antimatter asymmetry generated in the standard model is too small to yield anything like the observed asymmetry. The effect of the $\mathrm{CP}$ violation in the standard model from the Kobayashi-Maskawa (KM) phase is much too small[F.1]. In addition, in order to protect the asymmetry so that it can survive until the present, the Higgs mass cannot be heavier than $\mathrm{O}(40 \mathrm{GeV})$. This limit lies below the present experimental [F.1] There are some interesting proposal by Shaposhnikov and his collobarator to enhance the $\mathrm{CP}$ violation in the standard model at high temperature[5]. 
lower bound $m_{H} \geq 60 \mathrm{GeV}$. By these considerations, baryogenesis has an appealing implication of new physics beyond the standard model.

Assuming the existence of new physics at the $\mathrm{TeV}$ scale, we examine in this paper its impacts on electroweak baryogenesis[F.2] in terms of an effective lagrangian.

As the structure of the underlying theory beyond the standard model is presently unknown, it is useful to parametrize the effects of new physics in terms of effective lagrangian. In the literature, two schemes based on linear and nonlinear realization of $S U_{L}(2) \times U_{Y}(1)$ are used to construct the effective lagrangian. The nonlinear scheme, which puts less constraints on the effective lagrangian than the linear scheme does, allows more operators and provides more information for experiment to test. However, the effective lagrangian in the nonlinear scheme is built on the broken phase of $S U_{L}(2) \times$ $U_{Y}(1)$, so it is not useful for baryogenesis, which need to consider the change of vacuum expectation value of the Higgs field with temperature. We will restrict ourselves to the linear scheme.

In the linear scheme, the effects of new physics are described by higherdimension operators $O^{i}$,

$$
\mathcal{L}^{\text {new }}=\Sigma_{i} \frac{c_{i}}{\Lambda^{d_{i}-4}} O^{i},
$$

where $d_{i}$ are integers greater than 4 . The operators $O^{i}$ which have dimension $[\text { mass }]^{d_{i}}$ are $S U_{L}(2) \times U_{Y}(1)$ gauge invariant and contain only the standard model fields. The parameters $c_{i}$, determining the strength of the contribution [F.2] Other scenarios for baryogenesis can be found in Refs.[6, 8]. 
of operator $O^{i}$, can in principle be calculated by matching the effective theory with the underlying theory. However, they are taken as free parameters here since we don't know the underlying theory.

First of all, let us comment on the Higgs mass limit in this model. In a recent paper[7], it has been demonstrated that in the effective theory the Higgs mass can be relaxed to the present experimentally allowed region. The arguments is straightforward: in the effective lagrangian, higher dimension operators change the relation between the Higgs mass and the quartic coupling of the Higgs field. Consequently, the requirement of avoiding washout of the baryon asymmetry is described as followes,

$$
m_{H}^{2}<(40 G e V)^{2}+8 \frac{v^{4}}{\Lambda^{2}},
$$

where the number in bracket corresponds to the approximate Higgs mass in the limit of $\Lambda \rightarrow \infty$; and $v \sim 250 \mathrm{GeV}$ is the vacuum expectation value of Higgs field. Equation (2) implies the $\Lambda \leq O(4 \mathrm{TeV})$. Certainly, the high energy scale of $\Lambda \sim 1 \mathrm{TeV}$, which is required to solve the hierarchy problem, allows an Higgs mass which satisfies comfortably the experimental lower bound of $60 \mathrm{GeV}$. Thus an indication of electroweak baryogenesis is that Higgs may be discovered at LEP II.

Having solved the "Higgs mass limit" problem we now consider the impacts of new $\mathrm{CP}$ violation required to produce enough baryon asymmetry on the electric dipole moments of electron and neutron. Firstly, we give a brief summary of electroweak baryogenesis[8].

The baryon asymmetry is related to the total change of the Chern-Simon 
number during the electroweak phase transition:

$$
\frac{\alpha_{W}}{4 \pi} \int_{t_{c}}^{\infty} d^{4} x W_{\mu \nu} \tilde{W}^{\mu \nu}
$$

where $\tilde{W}^{\mu \nu}=\frac{1}{2} \epsilon^{\mu \nu \lambda \rho} W_{\lambda \rho}$, and $t_{c}$ corresponds to the time when electroweak phase transition startes.

To have a net change of the Chern-Simon number, one needs a term breaking $\mathrm{P}$ and $\mathrm{CP}$. The simplest operator is[F.3]

$$
O_{w}=c_{w} \frac{g^{2}}{8 \pi^{2}} \frac{\phi^{2}}{\Lambda^{2}} \operatorname{Tr} W_{\mu \nu} \tilde{W}^{\mu \nu},
$$

where $\phi$ is the neutral component of the standard model Higgs doublet, $c_{w}$ a free parameter. Simply one can see that this operator induces a "chemical potential" $\mu=\frac{d}{d t}\left(c_{w} \frac{\phi^{2}}{\Lambda^{2}}\right)$ for the Chern-Simon number, with which the sphaleron process is biased. We have

$$
\begin{aligned}
n_{B} & =3 \int \frac{\Gamma_{s p h}}{T} \mu d t \\
& =3 \int \frac{\Gamma_{s p h}}{T} d\left(c_{w} \frac{\phi^{2}}{\Lambda^{2}}\right),
\end{aligned}
$$

where the factor 3 is the number of generations, $\Gamma_{s p h}$ the sphaleron rate.

During electroweak phase transition, Higgs field rolls from false vacuum $(\phi=0)$ down to the true vacuum $(\phi \neq 0)$. Through $\mathrm{CP}$ violating operator $O_{w}$ the sphaleron process is biased, resulting in net baryon asymmetry. The final asymmetry can be obtained by integrating Eq.(5). However, $\Gamma_{s p h}$ in the integral is not well known. Instead, what we know about $\Gamma_{s p h}$ are:

[F.3] This operator has been considered in $\operatorname{Ref}[9]$. Here we use an equivalent, but slightly different, method to derive baryon asymmetry. 
i) In the unbroken phase

$$
\Gamma_{s p h}=\kappa\left(\alpha_{W} T\right)^{4}
$$

where $\kappa$ subsumes our ignorance about the exact rate, which is taken to be $0.1 \sim 1$ from numerical simulation[10].

ii) In the broken phase

$$
\Gamma_{s p h}=\gamma\left(\alpha_{W} T\right)^{-3} M_{W}^{7} e^{-\frac{E_{s p h}}{T}},
$$

where $E_{s p h} \sim \frac{g \phi(T)}{\alpha_{W}}$ and $\gamma$ a constant evaluated numerically in Ref.[11]. iii) $\Gamma_{s p h}$ in Eq.(5) will be much suppressed when the Higgs vacuum expectation value exceeds the critical value $\phi_{c}(T)$. In Ref.[12], it is estimated that $\phi_{c}(T) \sim 14 \frac{\alpha_{W} T}{g}$. There are also some arguments for smaller $\phi_{c}(T)[13]$.

So practically, the integral can be estimated by the following approximation: inserting a step function $\Theta\left(\phi_{c}-\phi\right)$ in the integrant and taking $\Gamma_{s p h}$ to be $\kappa\left(\alpha_{W} T\right)^{4}$. Now we arrive at the final ratio of baryon number density to entropy:

$$
\begin{aligned}
\frac{n_{B}}{s} & =\frac{3 \times 45 \times 0.5}{2 \pi^{2} g_{*}} \kappa c_{w} \alpha_{W}^{4}\left(\frac{T}{\Lambda}\right)^{2} \\
& \sim 4 \times 10^{-2} \kappa c_{w} \alpha_{W}^{4}\left(\frac{T}{\Lambda}\right)^{2},
\end{aligned}
$$

where $g_{*} \sim O(100)$ is the statistical weight factor appearing in the formula of entropy $s$ and $T \sim O(100 \mathrm{GeV})$ for weak phase transition. Taking $\Lambda \sim 1 T e V$, one can see that to explain the experimental value

$$
\frac{n_{B}}{s} \sim(0.4-1.4) \times 10^{-10},
$$


it is required that for $\kappa \sim 1-0.1$

$$
c_{w} \geq 0.1-1
$$

which is not an unreasonable value to expect.

Let us calculate the electric dipole moments of electron and neutron induced by $O_{w}$. At zero temperature, $\phi=\frac{1}{\sqrt{2}}\left(\begin{array}{c}0 \\ v+H\end{array}\right)$, with $\mathrm{H}$ being the standard Higgs particle. After diagonalizing the mass matrix of $W_{\mu}^{3}$ and $B_{\mu}$, one can see that an effective Higgs-photon-photon vertex is induced by Eq. (4):

$$
\mathcal{L}_{H \gamma \gamma} \sim c_{w} \frac{\alpha_{e m}}{32 \pi} \frac{H}{v} F_{\mu \nu} \tilde{F}^{\mu \nu}
$$

where $F_{\mu \nu}$ is the field strength of $U_{e m}(1)$. In Eq.(11) we have taken $\Lambda \sim$ $1 T e V$. This operator breaks $\mathrm{CP}$ and contributes to electric dipole moments of the fermion, which can been seen by attaching the Higgs and one photon to the fermion line[14]. We use dimensional regularization to regularize this divergent integration. Since the fundamental theory is assumed to be renormalizable, the infinities should be absorbed in the counterterms appearing in the effective lagrangian

$$
\frac{m_{\psi}}{\Lambda^{3}} i \overline{\Psi_{L}} \phi \sigma_{\mu \nu} \Psi_{R} F^{\mu \nu}+h . c
$$

where $m_{\psi}$ is the mass of fermion field $\Psi$.

The inclusion of such counterterm would introduce a free parameter as to preclude predictive power. Instead, what has been done in the literature is to focus on certain "Log-enhanced" term. Practically, we compute the leading correction of operator $O_{w}$ to the electric dipole moments of fermion in $\overline{M S}$ 
scheme, setting the counterterm in (12) to zero for $\mu$, the renormalization scale, to be $\Lambda$. We have now

$$
\frac{d_{\psi}}{e} \sim Q_{\psi} \frac{c_{w} \alpha_{e m} m_{\psi}}{32 \pi v^{2}} \frac{1}{(4 \pi)^{2}}\left\{\frac{1}{2}+2 \int_{0}^{1} d x \int_{0}^{1-x} d y \ln \frac{\Lambda^{2}}{m_{H}^{2} y+x^{2} m_{\psi}^{2}}\right\}
$$

where $Q_{\psi}$ is the electric charge of field $\Psi$. In this formula, $c_{w}$ is fixed as shown in Eq.(10) and $m_{H}$ is bounded from above as we mentioned before. Thus we have for electron and neutron

$$
\begin{aligned}
& \frac{d_{e}}{e} \geq 10^{-28}-10^{-27} \mathrm{~cm}, \\
& \frac{d_{n}}{e} \geq 10^{-27}-10^{-26} \mathrm{~cm} .
\end{aligned}
$$

In the derivation of the electric dipole moment of neutron we have used nonrelativistic quark model, where $d_{n} \sim d_{d}, d_{u}$. Clearly the predicted values in Eqs.(14) are very close to the present experimental limits.

In conclusion, we have demonstrated that a solution to the hierarchy problem of the Higgs sector of the standard model opens the possibility for electroweak baryogenesis. We have predicted the electric dipole moments of electron and neutron from the new $\mathrm{CP}$ violation term required to produce enough baryon asymmetry of the universe. We should point out that predictions of the electric dipole moments from baryogenesis also exist in two Higgs model[15], minimal supersymmetric standard model[16] and left-right symmetric model[17]. However, the effective lagrangian approach to electroweak baryogenesis used in this article depends only on the general form of operators available at low energies, and can describe theories in which 
no exotic particles appear at low energies. Examples of this kind of theory include certain "composite Higgs" models[18] and composite models[19].

One may wonder why we choose among many possible operators only the particular one given in Eq. (4) for baryogenesis. If one assumes that the new physics couples very weakly to quarks and leptons, and contributes mainly to the gauge bosons' propagator corrections (the so-called "oblique corrections")[20], there are only two operators of the lowest dimension which are relevent to electroweak baryogenesis:

$$
\begin{aligned}
O_{w} & \sim \frac{\phi^{2}}{\Lambda^{2}} \operatorname{Tr} W_{\mu \nu} \tilde{W}^{\mu \nu}, \\
O_{g} & \sim \frac{\phi^{2}}{\Lambda^{2}} \operatorname{Tr} G_{\mu \nu} \tilde{G}^{\mu \nu},
\end{aligned}
$$

where $G_{\mu \nu}$ is the gluon field strength. The $O_{g}$ is important only in the case of a thick enough bubble wall so that QCD anomalous interaction is in thermal equilibrium[21].

To conclude, we would like to point out that the electroweak baryogenesis calculations available so far are qualitative and the quatitative results obtained are probably only accurate to within a couple of order of magnitude. Thus future low energy measurements on Higgs mass, $d_{e}$ and $d_{n}$ will test the present knowledge about electroweak baryogenesis.

X.Z. would like to thank R.N. Mohapatra and R.D. Peccei for helpful discussions. The work of XZ is supported by a grant from National Science Foundation. BLY is suppport in part by the U.S. Department of Energy 
under contract No. W-7405-Eng-82, Office of Energy Research (KA-01-01), and Division of High Energy and Nuclear Physics.

\section{References}

[1] S. Weinberg, Phys. Rev. Lett. 19, (1967) 1264; A. Salam, in Elementary Particle theory: relativistic groups and analyticity, Nobel Symp. No. 8, ed. N. Svartholm (Almqvist and Wiksell, Stockholm, 1969) p. 367; S.L. Glashow, J. Iliopoulos and L. Maiani, Phys. Rev. D2, (1970) 1285.

[2] P. Langacker, UPR-0555T, March 24, 1993.

[3] A. Sakharov, JETP lett. 5 (1967) 24.

[4] V. Kuzmin, V. Rubakov and M. Shaposhnikov, Phys. Lett. B155, (1985) 36.

[5] M. Shaposhnikov, Phys. Lett. B277 (1992) 324; Erratum, Phys. Lett. B282, (1992) 483; G.R. Farrar and M.E. Shaposhnikov, Phys. Rev. Lett. 70, (1993) 2833.

[6] A. Dolgov, Phys. Rept. 222 (1990) 222.

[7] X. Zhang, Phys. Rev. D47, (1993) 3065.

[8] A. Cohen, D. Kaplan and A. Nelson, UCSD-PTH-93-02, BUHEP-93-4, January 1993 and references therein.

[9] M. Dine, P. Huet, R. Singleton and L. Susskind, Phys. Lett. B257, (1991) 351.

[10] J. Ambjorn, T. Askgaad, H. Porter and M. Shaposhnikov, Phys. Lett. B244, (1990) 479 . 
[11] L. Carson, X. Li, L. McLerran and R. Wang, Phys. Rev. D42 (1990) 2127.

[12] B. Liu, L. McLerran, N. Turok, Phys. Rev. D46, (1992) 2668.

[13] M. Dine, P. Huet and R. Singleton, Nucl. Phys. B375 (1992) 625.

[14] S. Barr and A. Zee, Phys. Rev. Lett. 65, (1990) 21.

[15] A. Kazarian, S. Kuzmin and M. Shaposhnikov, Phys. Lett. B276, (1992) 131.

[16] A. Cohen and A. Nelson, Phys. Lett. B279, (1992) 111.

[17] R. Mohapatra and X. Zhang, Phys. Rev. D46, (1992) 5331.

[18] H. Georgi, Lectures at les Houches Summer School (1985) and references therein.

[19] R.D. Peccei, in the proceeding of The 1987 Lake Louise Winter Institute, Selected Topics in Electroweak Interaction, J.M. Cameron et al., ed (World Scientific, 1987) and references therein.

[20] B.W. Lynn, M.E. Peskin and R.G. Stuart, in Physics at LEP, ed. J. Ellis and R. Peccei, CERN 86-02 (1986)I, 90; D.C. Kennedy and B.W. Lynn, Nucl. Phys. B332, (1989) 1; M.E. Peskin and T. Takeuchi, Phys. Rev. Lett. 65 (1990) 964.

[21] R. Mohapatra and X. Zhang, Phys. Rev. D45, (1992) 2699. 